\title{
Spatial Co-Location Pattern Discovery from Fuzzy Objects
}

\author{
Zhiping Ouyang, Lizhen Wang* and Pingping Wu \\ Department of Computer Science and Engineering \\ School of Information Science and Engineering \\ Yunnan University, Kunming 650091, China \\ *lzhwang@ynu.edu.cn
}

\author{
Received 27 April 2014 \\ Accepted 19 October 2016 \\ Published 10 March 2017
}

\begin{abstract}
A spatial co-location pattern is a group of spatial objects whose instances are frequently located in the same region. The spatial co-location pattern mining problem has been investigated extensively in the past due to its broad range of applications. In this paper we study this problem for fuzzy objects. Fuzzy objects play an important role in many areas, such as the geographical information system and the biomedical image database. In this paper, we propose two new kinds of co-location pattern mining for fuzzy objects, single co-location pattern mining $(\mathrm{SCP})$ and range co-location pattern mining $(\mathrm{RCP})$, to mining co-location patterns at a membership threshold or within a membership range. For efficient SCP mining, we optimize the basic mining algorithm to accelerate the co-location pattern generation. To improve the performance of RCP mining, effective pruning strategies are developed to significantly reduce the search space. The efficiency of our proposed algorithms as well as the optimization techniques are verified with an extensive set of experiments.
\end{abstract}

Keywords: Spatial data mining; co-location patterns; fuzzy objects.

\section{Introduction}

A spatial co-location pattern represents a group of spatial objects whose instances are frequently located in a spatial neighborhood. Mining spatial co-location patterns is the discovery and excavation of the relationship between spatial objects in spatial databases. Spatial co-location patterns yield important insights for many applications. For example, a mobile service provider may be interested in mobile service patterns frequently requested by geographically neighboring users. The frequent neighboring request set can be used for providing attractive location-sensitive advertisements. Other application

This is an Open Access article published by World Scientific Publishing Company. It is distributed under the terms of the Creative Commons Attribution 4.0 (CC-BY) License. Further distribution of this work is permitted, provided the original work is properly cited.

* Corresponding author 
domains include Earth science, public health, transportation, biology, etc. After an extensive study on this topic during the last decades, numerous algorithms have been proposed. ${ }^{1-7}$ Recently, with the proliferation of mobile computing, spatial co-location patterns have also been extended to moving object databases and uncertain databases. To the best of our knowledge, all these work assume the underlying data to be crisp objects, which means their compositions and boundaries are deterministic. However, in some real applications such as biomedical image analysis and geographical information systems (GIS), the data may not satisfy this assumption. ${ }^{21}$

In real life, such as "old man", "tall tree", etc, the boundary of these objects cannot be identified easily, these objects are known as fuzzy objects. Although fuzzy objects have long been studied in GIS community, ${ }^{23,24}$ spatial co-location patterns mining still remain uninvestigated. To address the problem of spatial co-location pattern mining in Euclidean space over large fuzzy datasets, we propose single co-location pattern mining (SCP) by mining co-location patterns at a membership threshold. Furthermore, to make sure no valuable information is discarded, let users try different membership threshold to examine the differences of the results, we generalize SCP mining by replacing single membership thresholds with a range of membership threshold.

To sum up, we make the following major contributions:

- We define FPR and FPI, The key difference from traditional definitions is that it takes a probability value to improve accuracy. Moreover, we used the $f$ threshold and d_threshold, as the parameters so that users can control the confidence level based on which the co-location patterns is evaluated.

- Two new kinds of co-location patterns mining on fuzzy objects are presented, single co-location patterns mining (SCP) and range co-location patterns mining (RCP), to mining co-location patterns at a membership threshold or within a membership range.

- We propose efficient algorithms to answer SCP and RCP, effective heuristic rules, including pruning most disqualifying objects, reducing the number of mining and narrowing the excavation, etc, are developed to improve the efficiency of co-location pattern mining.

- Extensive experiments are conducted to verify that the proposed algorithms as well as their optimization techniques achieve satisfactory performance.

The remainder of this paper is organized as follows. In Section 2, we discuss the related work. The concepts and lemmas are given in Section 3. Proposed algorithms for answering SCP (single co-location patterns) and RCP (range co-location patterns) mining are presented in Sections 4 and 5. We show the experiment results in Section 6. Finally we conclude the paper in Section 7.

\section{Related Works}

There is a large body of research works on co-location pattern mining in spatial databases. The research on certain data is the most common type of co-location pattern mining and there are a lot of algorithms, such as the join-based algorithm, ${ }^{1,2}$ the partial- 
join and the join-less algorithm, ${ }^{3}$ the CPI-tree algorithm, ${ }^{4}$ the density-based algorithm, ${ }^{5}$ the order-clique-based algorithm, ${ }^{6}$ etc. Literatures ${ }^{1,2}$ define the related concepts of spatial co-location pattern mining problem, including $R$-neighbor, spatial co-location pattern, row instance, table instance, participation ratio (PR), participation index (PI) and colocation rule, etc. The research on uncertain data is more and more, such as the spatial colocation mining on interval data, ${ }^{8}$ the probabilistic prevalent co-location mining in spatially uncertain data sets, ${ }^{9}$ the prevalent co-location mining from spatially uncertain data whose locations are described by probability density function (PDF), ${ }^{10}$ Mining colocations from spatially uncertain data with probability intervals, ${ }^{11}$ the top-k probabilistic prevalent co-locations by grouping the possible worlds with matrices in uncertain data. ${ }^{12}$

Recently domain-driven pattern discovery has been attracting more researchers. Reference 13 integrates the spatial instance distribution information into prevalence metrics. The concept and mining methods of spatial high utility co-location patterns are presented in Refs. 14 and 15 studies the incremental mining of high utility co-location patterns. Reference 16 proposes a novel algorithm to mine causal rules from prevalent co-locations based on spatio-temporal databases. A framework for mining regional colocation patterns is proposed in Ref. 17. A spatial co-location pattern ordering approach is proposed in Ref. 18.

The concept of fuzzy objects has not been introduced to spatial database until Altman ${ }^{19}$ used fuzzy set theoretic approach for handling imprecision in spatial databases. Afterwards, simple metric operations such as the area of a fuzzy region and the length of a fuzzy line were further developed in Ref. 20, in which only unary functions were considered. Based upon that, more advanced spatial queries such as the nearest neighbor query were discussed in Ref. 21. However, it remains largely untouched to answer the spatial data mining problem such as co-location pattern mining, which is addressed in this paper.

\section{Models and Mining}

In this section, we firstly describe the basic concepts of co-location pattern mining for fuzzy objects. Then, two new co-location pattern mining methods are proposed in order to meet different user purpose. Finally, we discuss a lemma of the mining problem.

\subsection{Fuzzy object model}

Fuzzy objects are usually modeled by fuzzy sets, ${ }^{22}$ which are characterized by their membership function $\mu: R^{d} \rightarrow[0,1]$, mapping any element in the object space to a real value $\mu(x)$ within the interval $[0,1]$. An element mapped to zero means that the member is not included in the fuzzy set, while one describes a fully included member. Values strictly in between characterize the fuzzy members. Fuzzy objects can be defined in continuous space if a continuous membership function is can be given. But in real applications, such a membership function is often not explicitly available due to the diversity of fuzzy objects. Besides, fuzzy objects identified from raster images are 
normally represented by pixels which are actually discrete points. For these reasons, we adopt a very general discrete form to model fuzzy objects.

Definition 1. A fuzzy object in d-dimensional space is represented by a set of probabilistic spatial point $A=\{\langle a, \mu(a)\rangle|\mu(a)\rangle 0\}$, where $A$ is a fuzzy object, $a$ is a instance, $\mu(a)$ is the membership value of $a$ which indicates the probability of a belonging to $A$.

Figure 1 shows a fuzzy object $A$ and $\mu(a 1)=0.6, \mu(a 2)=0.01$. As can be seen from the figure, the membership value of instance $a 2$ is very low, and then we want to exclude it in the calculation. Because in real life, we consider the instance with low membership value has little contribution. Such as elderly, it is assumed that the membership value of 80 -year-old is 0.9 , and 40-year-old is 0.02 . For 40 -year-old, it has little contribution for the elderly, so in some applications, we can exclude it, only concerned with the practical application instance. So, we have the following definitions.

Definition 2. Given a user-defined membership threshold $f_{-}$threshold, $A_{f_{-} \text {threshold }}=$ $\{a \mid \mu(a) \geq f$ threshold $\}$ indicates that the collections to satisfy the user-defined membership threshold. As shown in Fig. 1, $A_{0.2}=\{a 1\}$.

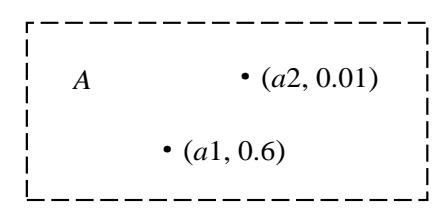

Fig. 1. Fuzzy object.

Given a set of spatial fuzzy objects $F$, a set of their instances $F I$, a spatial neighbor relationship $R$ over FI, In Fig. 2, there have 3 fuzzy objects $A, B, C$ and their instances. Instance $a 1$ stands for the first instance of $\mathrm{A}$. If $R$ is defined as a Euclidean distance metric and its threshold value is $d \_$threshold, two instances are neighbors if they satisfy the neighbor relationship:

$$
R(a 1, b 2) \Leftrightarrow \text { distance }(a 1, b 2) \leq d_{-} \text {threshold. }
$$

Given a subset of spatial instances $I=\{i 1, i 2, \ldots, i m\}, I \subseteq F I . I$ is called as an Rneighbor if $I$ forms a clique under the neighbor relation $R$.

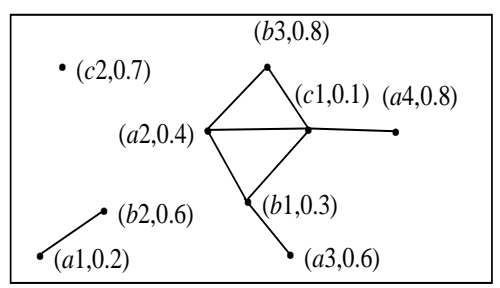

Fig. 2. Neighbor relationships. 
A spatial co-location pattern $c$ is a subset of spatial features, i.e., $c \subseteq F$. An Rneighbor $I$ is a row instance of a co-location pattern $c$ if $I$ contains instances of all the features in $c$ and no proper subset of it does so. The table instance of a co-location pattern $c$ is the collection of all row instances of $c$.

Definition 3. The fuzzy participation ratio $\operatorname{FPR}\left(c, f_{i}\right)$ of feature $f_{i}$ in a co-location $c=\left\{f_{1}, f_{2}, \ldots, f_{k}\right\}$ is a fraction, molecule is the membership value of all instances of $f_{i}$, where the instance in table instance, denominator is the number of instances of $f_{i}$. The fuzzy participation ratio can be computed as

$$
\operatorname{FPR}\left(c, f_{i}\right)=\frac{\sum 1 * \mu(a)}{\mid \text { table_instance }\left(\left\{f_{i}\right\}\right) \mid}
$$

where $a \in \pi_{f_{i}}$ (table_instance $\left.(c)\right), \pi$ is the relational projection operation with duplication elimination.

Definition 4. The fuzzy participation index $F P I(c)$ of a co-location $c=\left\{f_{1}, f_{2}, \ldots, f_{k}\right\}$ is the minimum in all $\operatorname{FPR}\left(c, f_{i}\right)$ of co-location $c$ :

$$
\operatorname{FPI}(c)=\min _{i=1}^{k} \operatorname{FPR}\left(c, f_{i}\right) .
$$

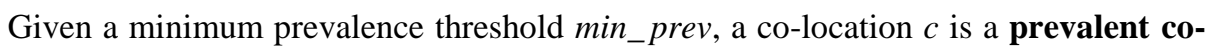

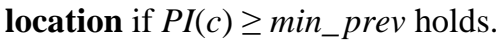

Example 1. In Fig. 2, there have three fuzzy objects, $A=\{(a 1,0.2),(a 2,0.4),(a 3,0.6)$, $(a 4,0.8)\}, B=\{(b 1,0.3),(b 2,0.6),(b 3,0.8)\}, C=\{(c 1,0.1),(c 2,0.7)\}$. Two instances are connected with a solid line if they are neighbors. Suppose co-location $c=\{A, C\}$ and min_prev $=0.2 . \operatorname{FPR}(c, A)=(1 * 0.4+1 * 0.8) / 4=0.3, F P R(c, C)=(1 * 0.1) / 2=0.05$, then $F P I(c)=\min \{0.3,0.05\}=0.05$, so $c$ is a non-prevalent co-location pattern.

Definition 4 (Description). Suppose fuzzy object $A$ have four instances, $a 1$ is a instance of $A$, Fig. 3 shows the relationship between FPR and the membership value of $a 1$. The abscissa is the membership, and the ordinate is the value of FPR. As can be seen from the figure, when the membership value is 0 , naturally, the $F P R$ is 0 , and when the membership value is 1 , the $F P R$ is 1 , like the traditional definition. As the membership value of instance $a 1$ increases, the $F P R$ will also increase accordingly.

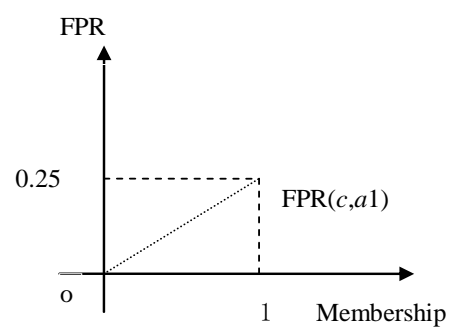

Fig. 3. Definition 4 description. 


\subsection{Co-location pattern mining}

In this subsection, we propose two new types of co-location pattern mining for fuzzy objects, i.e., SCP mining and RCP mining.

Definition 5 (SCP Mining). Given a fuzzy object dataset $F$, a set of their instances FI, d_threshold, min_prev and $f_{-}$threshold. SCP mining will get a set of prevalent colocation patterns.

The intuition behind this mining definition is to let users choose the $f$ threshold based on which the prevalent co-location patterns will be get. Furthermore, one can even try different $f_{-}$threshold to examine the differences of the results, to make sure no valuable information is discarded. Motivated by this application, we generalize SCP mining by replacing single $f_{-}$threshold with a range of $f_{-}$threshold, resulting in our second mining definition.

Definition 6 (RCP Mining). Given a fuzzy object dataset $F$, a set of their instances $F I$, a $f_{-}$threshold range $R=\left[a_{s}, a_{e}\right], d \_t h r e s h o l d$ and min_prev. RCP mining returns a set of prevalent co-location patterns $P=\left\{\left\langle c, R_{c}\right\rangle \mid R_{c} \in\left[a_{s}, a_{e}\right]\right\}$, where $c$ is a prevalent colocation pattern at any fuzzy threshold $f_{-}$threshold $\in R_{c}, R_{c}$ is called the qualifying range of $c$.

RCP mining is more powerful (yet more computationally challenging) since it allows the user to specify a range of $f_{-}$threshold and returns all the prevalent co-location patterns along with their qualifying ranges.

Example 2. Consider Fig. 4 where the FPI of two co-location patterns are shown. Let min_prev $=0.7$. If we set $f$ threshold to 0.4 , SCP mining will return the set $\{c 1\}$ as the result; but the set $\{c 1, c 2\}$ turns to be result if $f_{-}$threshold is changed to 0.5 . Furthermore, if the user issues a RCP mining with $R=[0.2,0.6]$, the result set should be $\{\langle c 1,[0.25,0.6]\rangle,\langle c 2,[0.2,0.35] \cup[0.45,0.6]\rangle\}$.

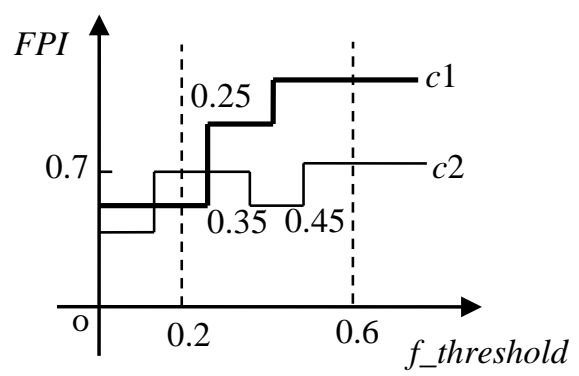

Fig. 4. Co-location pattern mining in RCP mining.

The new co-location pattern mining for fuzzy objects are quite different from the traditional ones such as certain data or uncertain data mining, in the sense that each object may be fully, partially or not involved at all in the mining evaluation, depending on the 
given membership threshold, which poses great challenges on the efficiency of mining algorithms. In the following Sections 4 and 5, we will try to overcome these difficulties and design efficient solutions to answer these mining.

\subsection{Lemma}

Lemma 1. The fuzzy participation ratio and the fuzzy participation index are monotonically non-increasing with increases in the size of the co-location.

Proof. Suppose a spatial instance is included in the table instances of co-location $c$. For co-location $c^{\prime} \subseteq c$, the spatial instance must be included in the table instances of $c^{\prime}$. The opposite is not true. Therefore, the fuzzy participation ratio is monotonically nonincreasing.

Because the fuzzy participation index is the minimum in all $F P R\left(c, f_{i}\right)$ of co-location $c$, when the size of the co-location increases, the fuzzy participation index is decreases, so it is also monotonically non-increasing.

\section{SCP Mining}

\subsection{Basic SCP mining}

The basic SCP mining uses the ideas of the Apriori-like algorithm to generate size- $k$ prevalent co-location patterns. Algorithm iteratively performs four steps: (1) Filters the set of candidate co-location patterns; (2) Calculates table instances set of candidate colocation patterns; (3) Pruning; (4) Generates co-location rules.

Step 1 is based on $f_{-}$threshold to obtain fuzzy set and their instance set, Step 2 for initialization, Step 3 to generate prevalent co-location pattern set, wherein, Step 3.1 for filtering the set of size- $k$ candidate co-location pattern, Step 3.2 to calculate size- $k$ table instances set of candidate co-location patterns set, Steps 3.3 and 3.4 to generate size- $k$ prevalent co-location pattern set and rule set.

\footnotetext{
Algorithm 1: Basic SCP mining

Input: $S F$ : spatial fuzzy objects set, $S F I$ : fuzzy instances set, min_prev: participation index threshold, min_conf: conditional probability threshold, d_threshold: distance threshold, $f$ threshold: membership threshold, $F, F I$ : fuzzy set and their instance set that satisfy the conditions of $f_{-}$threshold.
}

Output: FP: co-location rules set.

Variables: $k$ : co-location size, $C_{k}$ : a set of size- $k$ candidate co-location patterns, $T_{k}$ : a set of table instances of $C_{k}, P_{k}$ : a set of size- $k$ prevalent co-location patterns, $R_{k}$ : a set of size- $k$ co-location rules.

\section{Steps:}

$1 F, F I=$ gen_fdata $\left(S F, S F I, f \_\right.$threshold $)$;

$2 P_{1}=F, F P=\phi$; 
3 for $\left(k=2 ; P_{k-1} \neq \phi ; k++\right)$ do

$3.1 C_{k}=$ gen_candidate_co-location $\left(k, P_{k-1}\right)$;

$3.2 T_{k}=$ gen_table_instance $\left(C_{k}, T_{k-1}\right)$;

$3.3 P_{k}=$ sel_prev_co-location $\left(C_{k}, T_{k}\right.$, min_prev $)$;

$3.4 R_{k}=$ sel_co-location_rule $\left(P_{k}, T_{k}\right.$, min_conf $)$;

$3.5 F P \leftarrow F P \cup R_{k}$;

4 return $F P$.

\subsection{Pruning fuzzy objects}

Because the basic SCP mining algorithm requires a lot of distance calculation and join operations on fuzzy objects, so we should as much as possible to cut off some of the fuzzy objects that cannot exist in any prevalent co-location patterns. Based on the above considerations, the paper puts forward an effective pruning.

Lemma 2. For a fuzzy object $A$, if the maximum fuzzy participation ratio max_FPR is less than the minimum participation index threshold min_prev, then the fuzzy object A could not exist in any prevalent co-location pattern.

Proof. Reductio ad absurdum: (1) assumption of fuzzy object $A$ in a prevalent co-location pattern, then we can get $\operatorname{FPR}(c, A) \geq \min$ _prev. (2) The maximum fuzzy participation ratio is that it is all instances in table instances of $c$, according to the definition of fuzzy participation ratio, maximum fuzzy participation ratio is equal to the all instances of $A$ membership with the instances number ratio, by the condition of the lemma, it is less than the minimum participation index threshold min_prev, then we can get $F P R(c, A)<$ min_prev, contradicts the assumption. So the fuzzy object $A$ could not exist in any prevalent co-location pattern.

Example 3. In Fig. 2, let min_prev $=0.6$. Assumptions of all instances of object $C$ in the table instance of a co-location pattern $c$, then $m a x \_F P R=0.1 / 2+0.7 / 2=0.4<0.6$. By Lemma 2, object $C$ may not be found in any prevalent co-location pattern, so $C$ can be pruned.

Using the Lemma 2, the fuzzy objects can be preliminary pruning, reduce the time complexity of the algorithm. In Algorithm 2, Step 1 is the calculation of max_FPR.

\subsection{Reducing join}

Although the improved algorithm of Section 4.2 can reduce the computation between instances, but in the process of co-location pattern mining, there are still a large number of join operations, it is time-consuming. In the basic SCP mining algorithm, the first is to generate candidate patterns, and then according to the min_prev, the candidate patterns are pruned. Actually, in the beginning stages of creation of the table instance, we can cut some candidate patterns that cannot satisfy the condition of min_prev, avoid a lot unnecessary join operations between table instances. 
Algorithm 2: Pruning fuzzy objects

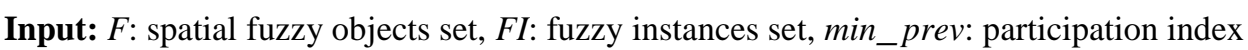
threshold, max_FPR: the maximum fuzzy participation ratio.

Output: $F, F I$.

Steps:

1 for all fuzzy object $f \in F$ do

1.1 compute max_FPR;

1.2 if $m a x \_F P R<m i n \_p r e v$ then

1.2.1 $F=F-\{f\} ; F I=F I-\{a\}$, where $a \in f ;$

2 return $F, F I$.

Lemma 3. Suppose fuzzy object A belongs to the co-location pattern $c$, if the instance of $A$ which in the table instance of $c$ satisfies $\max \{\mu(a)\}<\min \_$prev, then c can be pruned.

\section{Proof}

$$
\begin{aligned}
\text { FPR } c, A & \leq \frac{\sum_{i=1}^{n} \mu\left(a_{i}\right)}{\mid \text { table_instance }(\{A\}) \mid} \leq \frac{n * \max \left\{\mu\left(a_{i}\right)\right\}}{\mid \text { table_instance }(\{A\}) \mid} \\
& =\frac{n * \max \left\{\mu\left(a_{i}\right)\right\}}{n}=\max \left\{\mu\left(a_{i}\right)\right\} .
\end{aligned}
$$

So, when $\max \{\mu(a)\}<\min \_$prev, co-location pattern $c$ can be pruned.

Example 4. In Fig. 2, considering the size-2 co-location pattern $c=\{A, B\}$, each instance of object sequence according to the membership is non-incremental sort, assuming min_prev $=0.7$, in the neighbor relation $R$ of objects $\mathrm{A}$ and $\mathrm{B}, \max \{\mu(a)\}=0.6<$ min_prev, so the pattern $c$ can be pruned, and we do not need to calculate its table instance by joining operation.

In Algorithm 3, the variable max_flag is a flag, it determine whether the instance is the maximum membership, because the instances of objects sequence according to the membership is non-incremental sort, so the first join is the maximum ambiguity instances.

\subsection{Grid-based distance calculation}

In the traditional mining algorithms, compute the neighbor relationship need to calculate the distances between all instances, when the number of objects and the number of instances are very big, it will be very time-consuming. So in this section, we propose a grid-based distance calculation method, in which only the instances in the neighboring grids are considered when we calculate the distance between an instance and other instances. 
Algorithm 3: Reducing join

Input: $p 1_{k}, p 2_{k}$ : size-k prevalent co-location patterns, $t 1_{k}, t 2_{k}$ : table instance of $p 1_{k}, p 2_{k}$, $c 3_{k+1}$ : size- $k+1$ candidate co-location patterns.

Output: $t 3_{k+1}$ : table instance of $c 3_{k+1}$.

\section{Steps:}

1 max_flag $=$ true;

2 for $\left(I=1 ; I \leq t 1_{k}\right.$.count $\left.; i++\right)$

2.1 for $\left(j=1 ; j \leq t 2_{k}\right.$.count $\left.; j++\right)$

2.1.1 if $\left(\left(t 1_{k}[i]\right.\right.$. instance $_{1}=t 2_{k}[j]$.instance $1_{1} \ldots t 1_{k}[i]$. instance $e_{k-1}=t 2_{k}[j]$. instance $\left.k_{k-1}\right)$ and $\left(t 1_{k}[i]\right.$.instance $e_{k}, t 2_{k}[j]$.instance $\left.\left.e_{k}\right) \in R\right)$ then

2.1.1.1 if $\left(\right.$ max_flag $=$ true and $\left(t 1_{k}[i]\right.$.instance $<$ min_prev or $t 2_{k}[j]$.instance $<$ min_prev)) then

2.1.1.1.1 goto loop1;

2.1.1.2 else

$t 3_{k+1}=t 3_{k+1} \cup\left\{t 1_{k}[i]\right.$.instance join $t 2_{k}[j]$.instance $\} ;$

2.1.1.3 max_flag $=$ false ;

3 loop1: return $t 3_{k+1}$.

Definition 7. The neighboring grids of a grid are defined to including its own grid, down right, right, upper right and upper grids.

Example 5. In Fig. 5, the length of grid side is d_threshold, the shadow part is the neighboring grids of the instance $a 2$. When we compute the neighbor relationship of $a 2$, only compute the distance between $a 2$ and the instances in the shadow.

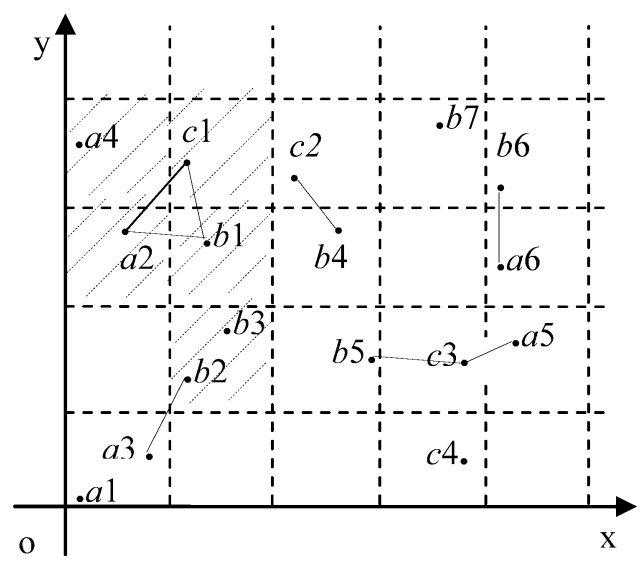

Fig. 5. Grid-based distance calculation. 
Algorithm 4 describes the calculation process of $R$ between instances.

Algorithm 4: Grid-based distance calculation

Input: $F$ : fuzzy objects; $F I$ : instances of $F$; $d_{-}$threshold: distance threshold.

Output: $R$ : the neighbor relationship.

Variables: $A$ : a fuzzy object; $a$ : instance of $A$; $d$ : the distance between two instances

\section{Steps:}

1 for each object $A \in F$;

1.1 for each instance $a \in A$;

1.1.2 compute $d$ between $a$ and $b$, where $b$ is in the neighboring grids of $a$;

1.1 .3 if $d<d \_$threshold then;

1.1.4 $a, b$ added to $R$;

2 return $R$.

\section{RCP Mining}

The SCP mining restricts the user to get the prevalent co-location set on a single $f \_$threshold. But sometimes, they may want to see how the prevalent co-location set varies on different $f_{-}$threshold. In such a case, they can issue an RCP mining where a f_threshold range instead of a single $f_{-}$threshold is accepted.

In this section we will introduce a basic RCP mining. Moreover, we develop several pruning rules to further improve the performance.

\subsection{Basic RCP mining}

To resolve the RCP mining, the most straightforward approach is to issue an SCP mining at every $f_{-}$threshold within the $f_{-}$threshold range $r$. In general, the membership value is an exact decimal. In this paper we assume membership value is accurate to two decimal places. So we set a variable $\varepsilon$, the value is 0.01 , assuming mining range $r=\left[a_{s}, a_{e}\right]$, when

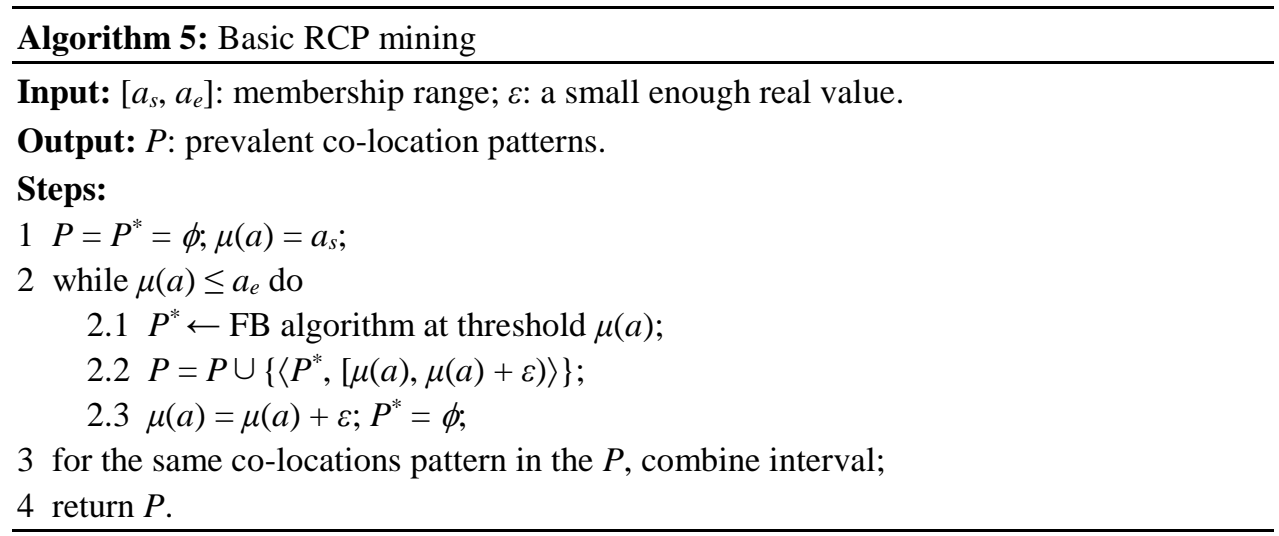


SCP mining is finished at $a_{s}$, another SCP mining is issued at $a_{s}+\varepsilon$. The above steps will repeat until it reaches $a_{e}$.

\subsection{Reducing mining number}

Before discuss the algorithm, we first introduce the concept of critical $f_{-}$threshold set.

Definition 8. Given a fuzzy set $F, r=\left[a_{s}, a_{e}\right]$, critical $f \_$threshold set $C(F)=\left\{\mu(a) \mid a_{s} \leq\right.$ $\left.\mu(a) \leq a_{e}, a \in F\right\} \cup\left\{a_{s}, a_{e}\right\}$, it is the membership values of all instances where the membership values belongs to $r$.

In Fig. 6, suppose $r=[0.1,0.6], \mu(a 1), \mu(a 3), \mu(a 4), \mu(b 1), \mu(c 2)$, and $\mu(d 1) \in r$, then $C(F)=\left\{\mu(a 1), \mu(a 3), \mu(a 4), \mu(b 1), \mu(c 2), \mu(d 1), a_{s}, a_{e}\right\}=\{0.1,0.2,0.3,0.6\}$.

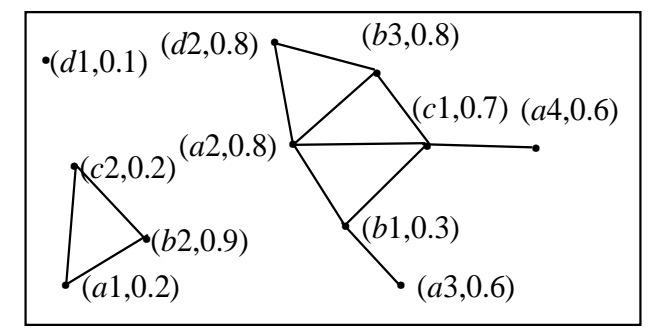

Fig. 6. Neighbor relationship.

Lemma 4. Suppose $\mu(a) \in C(F), \mu\left(a^{*}\right) \in C(F), \mu\left(a^{*}\right)>\mu(a)$, and $\mu\left(a^{*}\right)$ is adjacent to $\mu(a)$, if co-location pattern $c$ is prevalent at $f_{-}$threshold $\mu\left(a^{*}\right)$, then it is also prevalent at $r=\left(\mu(a), \mu\left(a^{*}\right)\right]$.

Proof. Because $\mu\left(a^{*}\right)$ and $\mu(a)$ adjacent, for any $f_{-}$threshold in $r=\left(\mu(a), \mu\left(a^{*}\right)\right]$, fuzzy participation ratio and fuzzy participation index will not be changed. So, when co-location pattern $c$ is prevalent at $f_{-}$threshold $\mu\left(a^{*}\right)$, then it is also prevalent at $r=\left(\mu(a), \mu\left(a^{*}\right)\right]$.

According to Lemma 4, the interval range $r$ is divided into some small intervals, and we only need to consider the boundary value of these intervals. So we can design a reducing mining number algorithm as follows.

According to $r=\left[a_{s}, a_{e}\right]$, obtained $C(F)$, for each value in $C(F)$, using SCP mining to mine co-location patterns, finally we can get the results.

Example 6. In Fig. 6, calculate the size-2 prevalent co-location patterns, assuming that $r=[0.1,0.6]$, min_prev $_{-} 0.4$, then $C(F)=\{0.1,0.2,0.3,0.6\}$. For each value in the $C(F)$, the algorithm use SCP mining to obtain a mining result, finally merging the mining results, obtained result set $P$, as shown in Table 1 . 
Table 1. Size-2 prevalent pattern result.

\begin{tabular}{cccccc}
\hline \multirow{5}{*}{ f_threshold } & $c 1=\{A, B\}$ & $c 2=\{A, C\}$ & $c 3=\{A, D\}$ & $c 4=\{B, C\}$ & $c 5=\{B, D\}$ \\
\cline { 2 - 5 } 0.1 & 0.4 & 0.4 & 0.2 & 0.45 & 0.27 \\
0.2 & 0.4 & 0.4 & 0.2 & 0.45 & 0.27 \\
0.3 & 0.37 & 0.6 & 0.27 & 0.37 & 0.27 \\
0.6 & 0.27 & 0.47 & 0.27 & 0.4 & 0.4 \\
\hline result $($ min_prev $=0.4)$ & $P=\{\langle c 1,[0.1,0.2]\rangle,\langle c 2,[0.1,0.6]\rangle,\langle c 4,[0.1,0.2] \cup(0.3,0.6]\rangle,\langle c 5,(0.3,0.6]\rangle\}$ \\
\hline
\end{tabular}

Algorithm 6: Reducing mining number

Input: $F$ : fuzzy objects; $F I$ : instances of $F ;\left[a_{s}, a_{e}\right]$ : membership range.

Output: $P$ : prevalent co-location patterns.

Variables: $C(F)$ : critical $f$ threshold set; $\mu(a), \mu\left(a^{*}\right)$ : ambiguity threshold; $P^{*}$ : prevalent co-location patterns

\section{Steps:}

$1 C(F)=P=P^{*}=\phi$;

$2 C(F)=$ get_critical_probability $\left(F, F I,\left[a_{s}, a_{e}\right]\right)$;

$3 P \leftarrow$ FB algorithm at threshold $C(F)[1]$;

4 for $(i=2 ; i \leq C(F)$.count; $i++)$

$4.1 \mu\left(a^{*}\right)=C(F)[i], \mu(a)=C(F)[i-1]$;

4.2 $P^{*} \leftarrow \mathrm{FB}$ algorithm at threshold $\mu\left(a^{*}\right)$;

$4.3 P=P \cup\left\{\left\langle P^{*},\left(\mu(a), \mu\left(a^{*}\right)\right]\right\rangle\right\}$;

$4.4 P^{*}=\phi$;

5 for the same co-locations pattern in the $P$, combine interval;

6 return $P$.

\subsection{Narrowing excavation area}

With the help of Lemma 4, the number of mining is significantly reduced. However, high computation cost still exists for the RCP mining, where we have to mine all prevalent colocation patterns for each value in $C(F)$. So our next goal is to narrow excavation area.

For the neighboring thresholds $\mu(a)$ and $\mu\left(a^{*}\right)$ in $C(F)$, in the basic RCP mining algorithm, when mining co-location patterns at $\mu(a)$ and $\mu\left(a^{*}\right)$, we use two SCP mining. In fact, after getting the result at $\mu(a)$, when we mine the result at $\mu\left(a^{*}\right)$, just need to find out the objects that the membership value is $\mu(a)$, and then judge whether these objects' patterns is prevalent, without computing all the patterns are prevalent by SCP mining.

Example 7. In Fig. 6, let $f_{-}$threshold range $r=[0.1,0.2]$, min_prev $=0.4$, then $C(F)=$ $\{0.1,0.2\}$. Considering Size-2 co-location patterns, when $f_{-}$threshold is 0.1 , as shown in Table 1, the prevalent patterns $P=\{c 1, c 2, c 4\}$. When compute the result at $f \_$threshold 
0.2 , because only $\mu(d 1)=0.1$, and $d 1 \in D$, so we just need to judge whether patterns $c 3=\{A, D\}, c 5=\{B, D\}$ is prevalent, without judging patterns $c 1, c 2, c 4$. Because according to Lemma 4 , the results of patterns $c 1, c 2, c 4$ remain unchanged, must be prevalent.

\section{Experiments}

In this section, we perform extensive experiments to verify the efficiency of the proposed methods and optimizations on both synthetic and real datasets. All the algorithms are implemented in C\# and run on a normal PC with core i7 $2.90 \mathrm{GHz}$ CPU and 8G memory.

\subsection{Datasets}

- For the synthetic dataset, we generate fuzzy objects and randomly distribute them into a $10000 * 10000$ space. Each object is a circle with radius of 0.5 and containing uniformly distributed points, whose membership values follow the two dimensional Gaussian distribution. In order to assure the kernel set is not null, we normalize the probability values across 0 to 1 .

- For the real dataset, we use a vegetation distribution data set of the Three Parallel Rivers of Yunnan Protected Areas. Similar with the synthetic dataset, we also normalize the probability values across 0 to 1 by using expert judge method.

For both datasets, 50 fuzzy objects are generated or selected. The all actual points are stored in files, and we index the fuzzy regions by R-tree. In the following experiments, we measure the number of prevalent patterns and running time of the algorithms under different parameter settings. Table 2 summarizes the parameters and their default values.

Table 2. Parameter settings.

\begin{tabular}{ccc}
\hline & \multicolumn{2}{c}{ Default Value } \\
\cline { 2 - 3 } Parameter & SCP Mining & RCP Mining \\
\hline number of instances $n$ & 100000 & 50000 \\
participation index threshold min_prev & 0.4 & 0.4 \\
distance threshold $d$ & 3000 & 2500 \\
membership threshold $f_{-}$threshold & 0 & - \\
f_threshold range $r$ & - & {$[0.1,0.4]$} \\
\hline
\end{tabular}

\subsection{SCP Mining performance evaluation}

In this subsection, we compare the performance of the basic SCP mining algorithm against its competitors, namely, SCP mining with pruning fuzzy objects (PO), PO with reducing join (PO_RJ), and PO_RJ with grid-based distance calculation (PO_RJ_GC). 


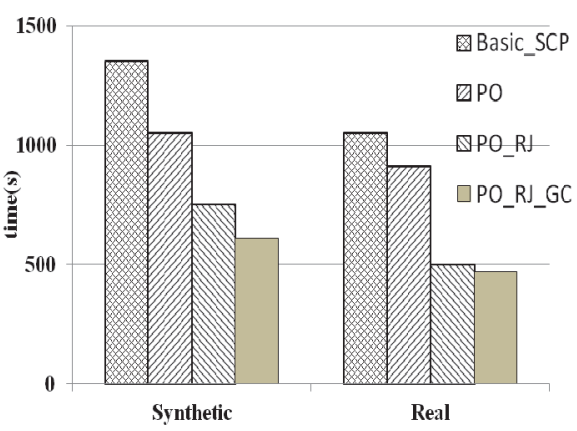

(a) Running time

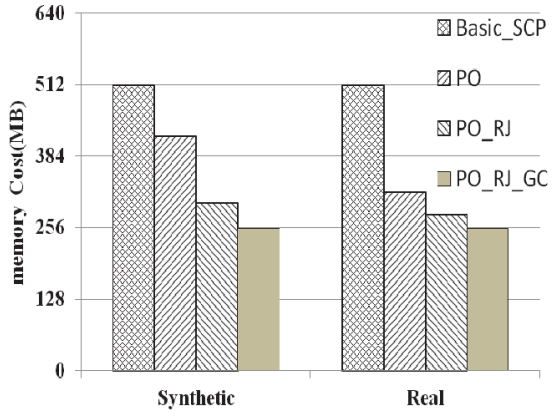

(b) Memory cost

Fig. 7. Effect of datasets.

\subsubsection{Effect of dataset}

First we run all algorithms on both datasets to see their running time and memory cost by using the default parameter settings. The results are shown in Figs. 7(a) and 7(b). As expected, the basic method exposes the worst performance on both datasets. More specifically PO and PO_RJ can avoid quite a few unnecessary object access and distance calculations. By further applying the grid-based distance calculation, PO_RJ_GC method achieves the best performance. As each algorithm shows similar performance on both datasets, we will just show the results on real datasets to keep our presentation concise.

\subsubsection{Effect of $n$}

First we study the impact of dataset size on the performance of SCP mining algorithms by varying the number of instances from 10000 to 200 000. As shown in Fig. 8(a), the run time of all algorithms increases because more $\mathrm{I} / \mathrm{O}$ operation is invoked and more distance need to evaluated. We also notice that the performance of all algorithms is still comparable when the dataset is relatively small, since the simple lower bound serves as a tolerable approximation when the data space is sparse. The advantages of the proposed optimizations become more obvious in larger dataset. From Fig. 9(a), all the algorithms generate more prevalent co-location patterns. This is because, when the number of instances grows, the density of whole data space becomes higher, which makes it more easy to generate prevalent co-location patterns. However, the number of prevalent colocation patterns of the traditional join-based algorithm is more than the PO_RJ_GC algorithm. This is because in the join-based algorithm, the participation ratio calculations do not consider the membership value, which leads to the participation ratio must be higher, in the same participation index threshold conditions, the number is more.

\subsubsection{Effect of min_prev}

We then compare the performance of all methods by varying min_prev from 0.8 to 0.2. According to Figs. 8(b) and 9(b), as the participation index threshold becomes lower, 


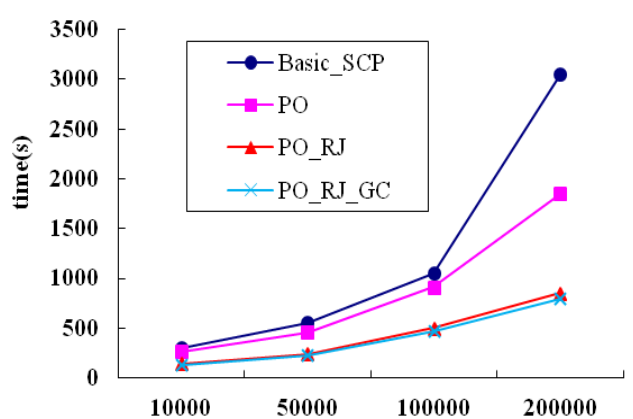

(a) Diff. $n$

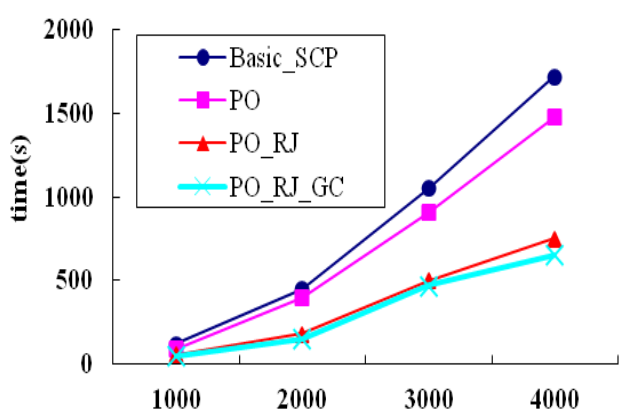

(c) Diff. $d$

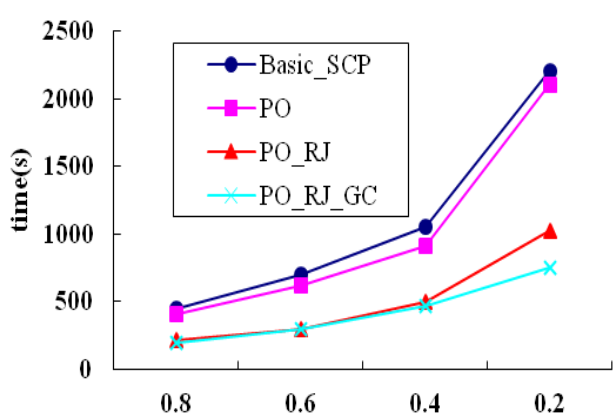

(b) Diff. min_prev $_{-}$

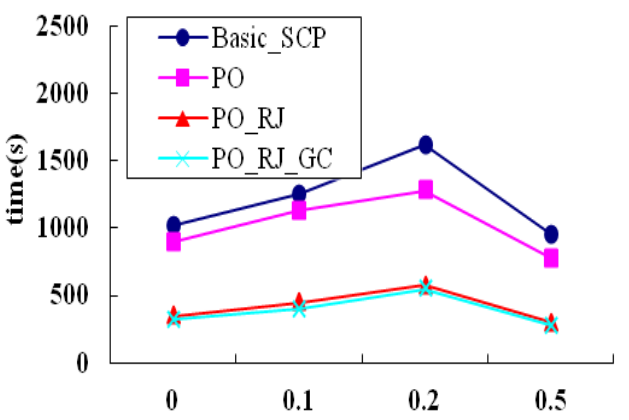

(d) Diff. f_threshold

Fig. 8. Running time of SCP mining.

more patterns satisfy the condition of min_prev, naturally, the performance of all algorithms deteriorate.

\subsubsection{Effect of $d$}

Next we study the impact of distance threshold $d$ on the performance of SCP mining algorithm by varying $d$ from 1000 to 4000 . The results are shown in Figs. 8(c) and 9(c). As distance threshold becomes large, the number of instance satisfied the $R$ relation will increase, lead to more running time. The number of prevalent co-location patterns also increase.

\subsubsection{Effect off_threshold}

We also study the impact of the membership threshold by varying $f_{-}$threshold from 0 to 0.5. From Figs. 8(d) and 9(d) we can observe that with the membership threshold become larger, the running time of all algorithms increase at first, but after 0.2 , the time began to decline, this is because in the beginning, when pruned low membership instances, the number of prevalent patterns will rise. But with the membership threshold becomes larger 


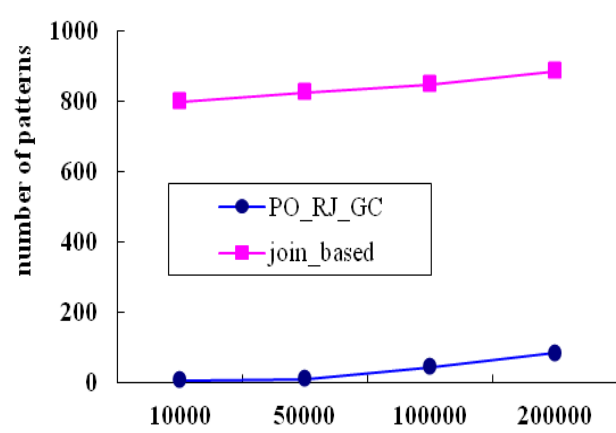

(a) Diff. $n$

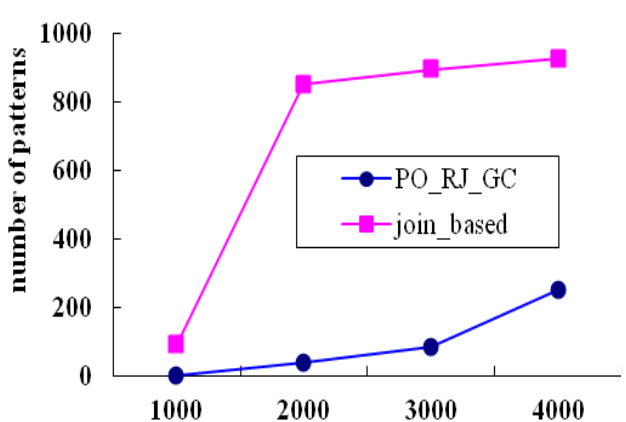

(c) Diff. $d$

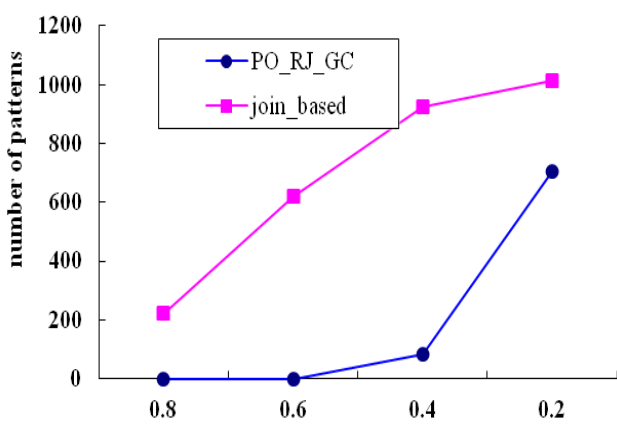

(b) Diff. min_prev

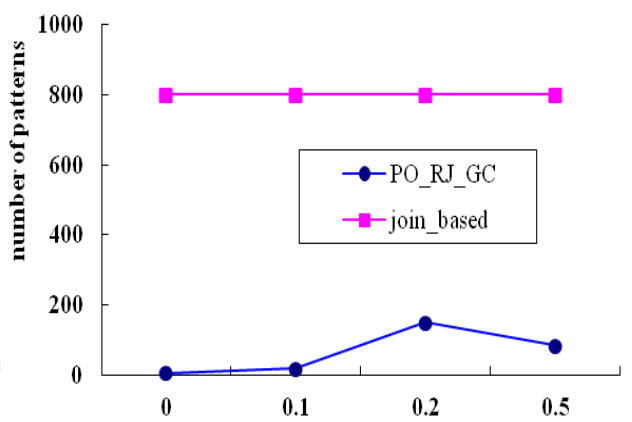

(d) Diff. $f$ threshold

Fig. 9. Mining results.

and larger, the pruned instances will be more and more, which means the number of prevalent patterns will be less and less, leading to the required time reduced.

\subsection{RCP mining performance evaluation}

To verify the efficiency of our proposed methods for answering RCP mining, we compare the performance of the basic algorithm with the optimized algorithms, i.e., basic $\mathrm{RCP}$ method with reducing mining number (RN) and RN with narrowing excavation area (RN_NA).

\subsubsection{Effect of $n$}

The first set of experiments tests the scalability of all RCP schemes by varying the dataset size from 5000 to 50 000. Results are shown in Fig. 10(a). From which we can see that performance of all algorithms degrade as the dataset grows, but the optimized algorithms are constantly superior to the basic method by at least one order of magnitude. Since the basic method relies on invoking lots of SCP mining procedure, it incurs high I/O and computation cost. With the performance getting worse in larger dataset, the basic RCP mining deteriorates even faster. 


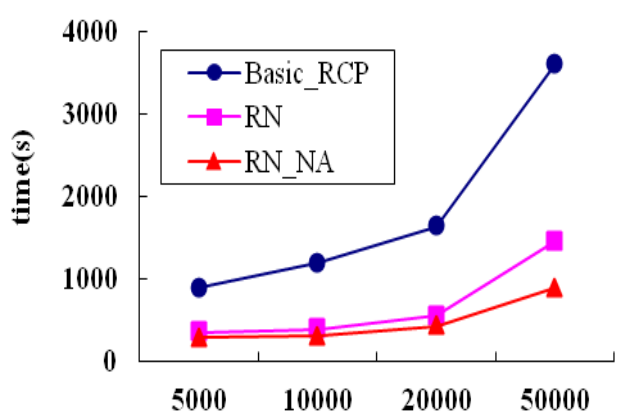

(a) Diff. $n$

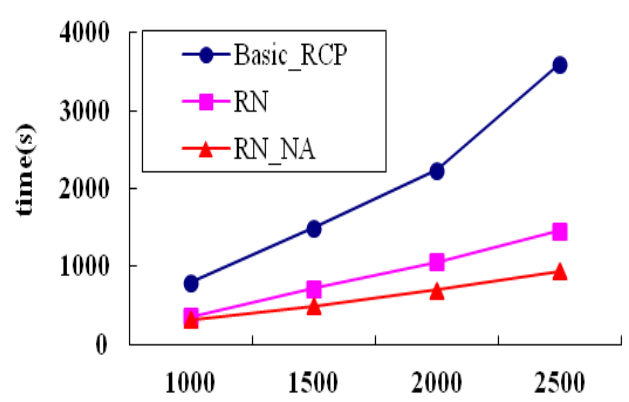

(c) Diff. $d$

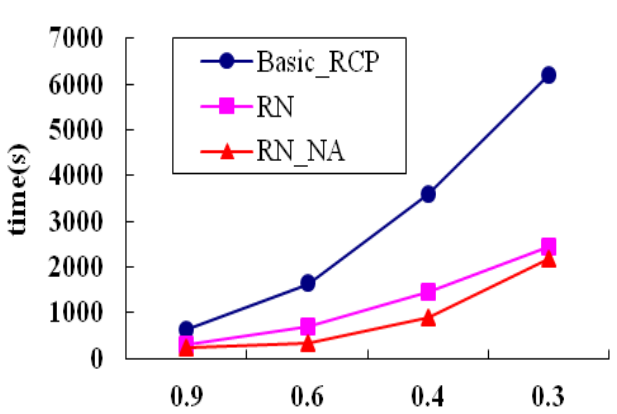

(b) Diff. min_prev

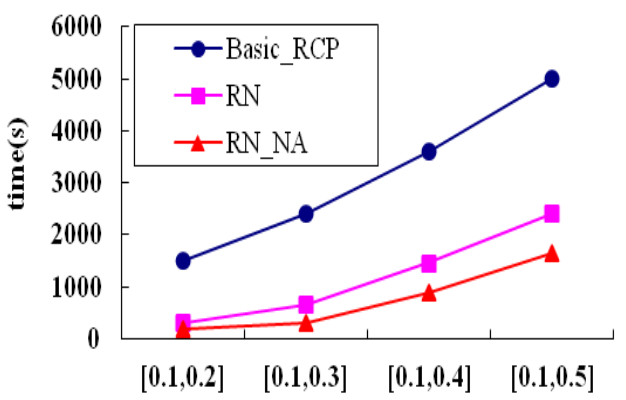

(d) Diff. $r$

Fig. 10. Running time of RCP mining.

\subsubsection{Effect of min_prev}

Then we study performance trend of all algorithms at different participation index threshold. Figure 10(b) clearly demonstrates the remarkable advantage of our optimizations, the cause of which is similar with the experiment 8(b) (Figure 8(b)). For all algorithms, their running time increases are caused by more distance to be computed as min_prev decreases.

\subsubsection{Effect of $d$}

We study the impact of the distance threshold by varying $d$ from 1000 to 2500 . As shown in Fig. 10(c), with $d$ increasing, the performance of the basic algorithm deteriorates very fast. This is because the SCP mining to generate more patterns and the basic_RCP algorithm needs to consider all the patterns.

\subsubsection{Effect of $r$}

Finally we examine the impact of different lengths of $f_{-}$threshold range on the performance of all algorithms by varying $r$ from [0.1, 0.2] to [0.1, 0.5]. Results are shown in Fig. 10(d), from which we can see that with the $f_{-}$threshold range becomes bigger, 
three algorithms for time increases. For the optimization algorithms, the rate of increase is more and more small, this is because when the membership threshold increases, the number of instances must be remove more, then the pattern number become fewer, although with the increase of the $f_{-}$threshold range, mining number will increase, but the time of the later mining will decrease, resulting in total time increased slowly.

\section{Conclusion}

Although mining co-location patterns is one of the most useful spatial data mining and fuzzy objects can often be found in many important applications, considering both has received rather limited attention. In this paper we have studied this problem in depth, defining two new types of co-location pattern mining for fuzzy objects. We have developed efficient algorithms to answer these mines. Extensive experiments have shown that our optimized algorithms achieve superior performance than to the baseline approaches constantly.

\section{Acknowledgments}

This research is supported by the National Natural Science Foundation of China (61472346, 61662086), the Natural Science Foundation of Yunnan Province (2015FB114, 2014FA023), the Program for Innovative Research Team in Yunnan University (XT412011), and the Research Foundation of the Educational Department of Yunnan Province (No. 2016ZZX304).

\section{References}

1. S. Shekhar and Y. Huang, Co-location rules mining: A summary of results, in Proc. of Int. Symp. on Spatial and Temporal Databases (SSTD) (CA, USA, 2001).

2. Y. Huang, S. Shekhar and H. Xiong, Discovering colocation patterns from spatial data sets: A general approach, IEEE Transactions on Knowledge and Data Engineering (TKDE) 16(12) (2004) 1472-1485.

3. J. S. Yoo and S. Shekhar, A join-less approach for mining spatial colocation patterns, IEEE Transactions on Knowledge and Data Engineering (TKDE) 18(10)(2006) 1323-1337.

4. L. Wang, Y. Bao, J. Lu and J. Yip, A new join-less approach for co-location pattern mining, in Proc. of the IEEE 8th Int. Conf. on Computer and Information Technology (CIT 2008) (Sydney, Australia, 2008), pp. 197-202.

5. X. Xiao, X. Xie, Q. Luo and W. Ma, Density based co-location pattern discovery, in SIGSPATIAL (California, USA, 2008), pp. 11-20.

6. L. Wang, L. Zhou, J. Lu and J. Yip, An order-clique-based approach for mining maximal colocations, Information Sciences 179 (2009) 3370-3382.

7. F. Qian, Q. He, K. Chiew and J. He, Spatial co-location pattern discovery without thresholds, Knowledge and Information Systems 33(2) (2012) 419-455.

8. L. Wang et al., Efficiently mining co-location rules on interval data, in Proc. of the 6th Int. Conf. on Advanced Data Mining and Applications (ADMA 2010), LNCS 6440 (Berlin: Springer, 2010), pp. 477-488. 
9. L. Wang, $\mathrm{P}$. $\mathrm{Wu}$ and $\mathrm{H}$. Chen, Finding probabilistic prevalent colocations in spatially uncertain data sets, IEEE Transactions on Knowledge and Data Engineering (TKDE) 25(4) (2013) 790-804.

10. Z. Liu and Y. Huang, Mining co-locations under uncertainty, in SSTD 2013 (Munich, Germany, 2013), pp. 429-446.

11. L. Wang et al., Mining co-locations from spatially uncertain data with probability intervals, in Proc. of the 14th Int. Conf. on Web-Age Information Management (WAIM 2013 Workshops), LNCS 7901 (Berlin: Springer, 2013), pp. 301-314.

12. L. Wang et al., Top-k probabilistic prevalent co-location mining in spatially uncertain data sets, Frontiers of Computer Science 10(3) (2016) 488-503.

13. C. Sengstock, M. Gertz and T. V. Canh, Spatial interestingness measures for co-location pattern mining, in Proc. of SSTDM (ICDM Workshop) (Brussels, Belgium, 2012), pp. 821826.

14. S. Yang, L. Wang, X. Bao and J. Lu, A framework for mining spatial high utility co-location patterns, in Proc. of the 12th Int. Conf. on Fuzzy Systems and Knowledge Discovery (FSKD 2015) (Zhangjiajie, China, 2015), pp. 631-637.

15. X. Wang, L. Wang, J. Lu and L. Zhou, Effectively updating high utility co-location patterns in evolving spatial databases, in Proc. of the 17th Int. Conf. on Web-Age Information Management (WAIM 2016) (Nanchang, China, 2016), Part I, LNCS 9658, pp. 67-81.

16. J. Lu, L. Wang and Y. Fang, Mining causal rules hidden in spatial co-locations based on dynamic spatial databases, in Proc. of the 2016 Int. Conf. on Computer, Information and Telecommunication Systems (CITS 2016) (Kunming, China, 2016), pp. 103-108.

17. S. Wang, Y. Huang and X. S. Wang, Regional co-locations of arbitrary shapes, in SSTD 2013 (Munich, Germany, 2013), pp. 19-37.

18. G. Yuan, L. Wang, P. Yang and L. Chen, Spatial co-location pattern ordering, in Proc. of the 2016 Int. Conf. on Computer, Information and Telecommunication Systems (CITS 2016) (Kunming, China, 2016), pp. 31-35.

19. D. Altman, Fuzzy set theoretic approaches for handling imprecision in spatial analysis, International Journal of Geographical Information Science 8(3) (1994) 271-289.

20. M. Schneider, Metric operations on fuzzy spatial objects in databases, in Proc. of the ACM Int. Symp. on Advances in Geographic Information Systems (ACMGIS 2000) (Washington, D.C., United States, 2000), pp. 21-26.

21. K. Zheng, P. C. Fung and X. Zhou, K-nearest neighbor search for fuzzy objects, in Proc. of the Special Interest Group on Management of Data (SIGMOD '10) (Indiana, USA, 2010), pp. 699-710.

22. L. Zadeh, Fuzzy sets, Information and Control 8(3) (1965) 338-353.

23. M. Schneider, Fuzzy topological predicates, their properties, and their integration into query languages, in Proc. of the ACM Int. Symp. on Advances in Geographic Information Systems (ACMGIS 2001) (New York, USA, 2001), pp. 9-14.

24. X. Tang and W. Kainz, Analysis of topological relations between fuzzy regions in a general fuzzy topological space, in Proc. of the Symposium on Geospatial Theory, Processing and Applications (Ottawa, Canada, 2002), pp. 114-123. 